\title{
Mutant Mitochondrial Elongation Factor G1 and Combined Oxidative Phosphorylation Deficiency
}

\author{
Marieke J.H. Coenen, M.Sc., Hana Antonicka, Ph.D., Cristina Ugalde, Ph.D., \\ Florin Sasarman, Ph.D., Rainer Rossi, M.D., J.G.A.M. Angelien Heister, B.Sc., \\ Robert F. Newbold, Ph.D., Frans J.M.F. Trijbels, Ph.D., \\ Lambert P. van den Heuvel, Ph.D., Eric A. Shoubridge, Ph.D., \\ and Jan A.M. Smeitink, M.D., Ph.D.
}

S U M MARY

From the Nijmegen Center for Mitochondrial Disorders (M.J.H.C., C.U., J.G.A.M.A.H. F.J.M.F.T., L.P.H., J.A.M.S.) at the Departments of Pediatrics (M.J.H.C., C.U. F.J.M.F.T., L.P.H., J.A.M.S.) and Human Genetics (J.G.A.M.A.H.), Radboud University Nijmegen Medical Center, Nijmegen, the Netherlands; Montreal Neurologica Institute and the Department of Human Genetics, McGill University, Montreal (H.A. F.S., E.A.S.); the Department of Pediatrics, Klinikum Neukölln, Berlin (R.R.); and Brune Institute of Cancer Genetics and Pharmacogenomics, Faculty of Life Sciences, Brunel University, Uxbridge, United King dom (R.F.N.). Address reprint requests to Dr. Smeitink at Nijmegen Center for Mitochondrial Disorders, Department of Pediatrics, University Medical Center Nijmegen $6500 \mathrm{HB}$ Nijmegen, the Netherlands, or at j.smeitink@cukz.umcn.nl.

Drs. Coenen and Antonicka contributed equally to this article.

N Engl J Med 2004;351:2080-6.

Copyright @ 2004 Massachusetts Medical Society. 


\section{CASE REPORTS}

The index patient, a female, was born of consanguineous Lebanese parents (first cousins) by cesarean section with intrauterine growth retardation and mild microcephaly. She was very stiff and had few spontaneous movements. From day 10 of life, profound metabolic acidosis was evident (blood lactate level, $17.1 \mathrm{mmol}$ per liter [normal, $<2.1 \mathrm{mmol}$ per liter]; lactate:pyruvate ratio, 38 [normal, 12 to 18]). Liver dysfunction, as measured by an elevation of direct bilirubin, was observed from day 7 and progressed to full liver failure on day 12 and death on day 27. Postmortem examination of the liver showed cholestasis and extensive necrosis. There was hypoplasia of the corpus callosum, and several symmetrical cystic lesions were present in the white matter in the area of the basal ganglia. The heart was normal, and a histologic examination of the skeletal muscle showed normal mitochondrial morphology and no ragged-red fibers. The activities of the oxidative phosphorylation complexes in fibroblasts were 40 percent, 69 percent, and 18 percent of the lowest control value for complex I, complex III, and complex IV, respectively. In muscle, complex I activity was 52 percent and complex IV activity was 54 percent of the lowest control value.

A male sibling of the index patient was born at 41 weeks of gestation. The maximal blood lactate level was $9.3 \mathrm{mmol}$ per liter, with a lactate:pyruvate ratio of 84. An ultrasonographic examination of the brain showed generalized atrophy and a small corpus callosum. The child had extremely delayed growth and development, as well as increased muscle tone in his upper extremities. Signs of liver failure were present at week 7 , leading to death at five months of age. The heart was normal. The activities of complex I and complex IV in fibroblasts were 13 percent and 31 percent of the lowest control value, respectively. Analysis of mtDNA by Southern blotting showed no rearrangements or reduction in mtDNA levels.

METHODS

\section{CONTROLS}

Samples from 100 ethnically matched controls were analyzed for restriction-fragment-length polymorphisms and showed a pattern that was homozygous for the wild-type allele.

\section{CELL CULTURE}

To extend the life span of cells, we immortalized primary human skin fibroblasts with a retrovirus containing the $E 6$ and $E 7$ genes of human papillomavirus type 16, or with E7 alone and a retroviral vector expressing the protein component of human telomerase reverse transcriptase. ${ }^{3}$ Skin fibroblasts from the siblings and controls were cultured in Dulbecco's modified Eagle medium supplemented with 10 percent fetal-calf serum, penicillin, and streptomycin. Samples were collected after informed consent was given.

\section{ENZYME MEASUREMENTS}

Complex I, complex III, complex IV, and citrate synthase activities were measured in fibroblast-cell extracts as described previously..$^{4,5} \mathrm{The} \mathrm{rho}^{\circ}$ test was performed, whereby $143 \mathrm{~B} . \mathrm{TK}^{-}$rho ${ }^{\circ}$ cells (cells without $\mathrm{mtDNA}$ that were derived from $143 \mathrm{~B} . \mathrm{TK}^{-}$cells) were fused with enucleated skin fibroblasts from the index patient and then selected in Dulbecco's modified Eagle medium containing 10 percent fetal-calf serum without pyruvate or uridine. Colonies were harvested for immunoblot analysis.

\section{CHROMOSOME TRANSFER}

The E6-and-E7-immortalized fibroblasts from the siblings were fused with human chromosomes isolated by microcell-mediated chromosome transfer $^{4,6}$ from a panel of mouse cell lines containing one extra human chromosome with a hygromycinresistant tag. ${ }^{7}$

\section{MICROSATELLITE MAPPING}

The short tandem-repeat microsatellite markers used for homozygosity and deletion mapping of chromosome 3 after chromosome transfer were derived from the Applied Biosystems ABI PRISM Linkage Mapping Set (version 2.5 MD-10). The intermarker distance was about $10 \mathrm{cM}$.

\section{CDNA SEQUENCING}

RNA was isolated from skin fibroblasts from the siblings and controls with the use of RNAzol. Primers were designed for the amplification of complementary DNA (cDNA) from MRPS22 and EFG1. The polymerase-chain-reaction (PCR) fragments were used for direct sequencing with the $A B I$ PRISM 377 DNA Sequencer (Applied Biosystems). 
ANALYSIS OF RESTRICTION-FRAGMENT-LENGTH POLYMORPHISMS

Genomic DNA was isolated from skin fibroblasts from the index patient and from blood of the parents, the sibling, and 100 controls. The DNA was amplified and digested with the restriction enzyme MseI.

BLUE-NATIVE POLYACRYLAMIDE-GEL ELECTROPHORESIS AND IMMUNOBLOTTING

Blue-native polyacrylamide-gel electrophoresis ${ }^{8}$ was used for separation of oxidative phosphorylation complexes on 5 percent to 15 percent polyacrylamide-gradient gels. Mitoplasts, which were prepared from fibroblasts by treatment with $0.8 \mathrm{mg}$ of digitonin per milligram of protein, were solubilized with 1 percent lauryl maltoside, and 10 to $20 \mu \mathrm{g}$ of the solubilized protein was used for electrophoresis. Complexes I through V were detected by immunoblot analysis with the use of monoclonal antibodies against complex III core protein 1 , the $\alpha$ subunit of complex $\mathrm{V}$, the $70-\mathrm{kD}$ subunit of complex II (Molecular Probes), monoclonal antibodies against complex IV subunit IV, and a polyclonal anti-ND1 antibody (an antibody acting against the mitochondrial-encoded ND1 subunit of complex I). For immunoblotting, fibroblasts were solubilized with 1.5 percent lauryl maltoside in phosphatebuffered saline, and $30 \mu \mathrm{g}$ of protein was separated by TRIS-Tricine sodium dodecyl sulfate-polyacrylamide-gel electrophoresis. The proteins were transferred to nitrocellulose, and antibodies directed against complex IV subunits II and IV and complex III core protein 2 were used for detection.

PULSE-LABELING OF MITOCHONDRIAL TRANSLATION PRODUCTS

In vitro labeling of mitochondrial translation was performed as previously described. ${ }^{9}$ Briefly, cells were labeled for 60 minutes at $37^{\circ} \mathrm{C}$ in methioninefree Dulbecco's modified Eagle medium containing $200 \mu \mathrm{Ci}$ per milliliter of [35S]methionine and $100 \mu \mathrm{g}$ per milliliter of emetine followed by $10 \mathrm{~min}-$ utes in regular Dulbecco's modified Eagle medium. Total cellular protein $(50 \mu \mathrm{g})$ was resuspended in loading buffer containing $93 \mathrm{mM}$ TRIS-hydrogen chloride ( $\mathrm{pH}$ 6.7), 7.5 percent glycerol, 3.5 percent sodium dodecyl sulfate, $0.25 \mathrm{mg}$ bromophenol blue per milliliter, and 3 percent mercaptoethanol, was sonicated for 3 to 8 seconds, and was loaded and run on 12 percent to 20 percent polyacrylamidegradient gels.

\section{CDNA CONSTRUCTS}

Retroviral vectors containing the cDNA sequence of three mitochondrial translation factors (EFG1, EFG2, and IF3) were created with the Gateway cloning system (Invitrogen). We used the reverse-transcriptase PCR (OneStep RT-PCR kit, Qiagen) to amplify cDNA from EFG2 and IF3 genes with the use of specific primers modified for cloning into Gateway vectors. The cDNA from EFG1 was amplified from IMAGE (Integrated Molecular Analysis of Genomes and Their Expression) clone 5574223 with use of specific primers modified for cloning into Gateway vectors. The PCR constructs were cloned into a Gateway-modified retroviral-expression vector, pLXSH. The fidelity of the cDNA clones was confirmed by automated DNA sequencing.

\section{INFECTION WITH VIRUSES}

Virus-producing cell lines were generated according to procedures described previously. ${ }^{10}$ Briefly, a human 293-derived retroviral packaging cell line (293GPG) was transfected with a retroviral construct containing one of the mitochondrial translation factors. Tetracycline was removed from the medium two days after transfection to induce virus production. Fibroblasts from the index patient and the controls were infected 72 to 96 hours later by exposure to virus-containing medium in the presence of $4 \mu \mathrm{g}$ per milliliter of polybrene as previously described. ${ }^{3}$

\section{GENBANK ACCESSION NUMBERS}

The GenBank accession numbers for EFG1, EFG2, IF3, and MRPS22 are NM_024996, NM_032380, AF410851, and NM_020191, respectively.

\section{RESULTS}

Fibroblasts from the index patient showed low levels of fully assembled complexes I, III, IV, and V but normal levels of complex II (Fig. 1). To determine whether this assembly defect was of nuclear or mitochondrial origin, we prepared transmitochondrial cytoplasmic hybrid (cybrid) cells by fusing enucleated fibroblasts from the index patient with rho ${ }^{0}$ cells, which are devoid of mtDNA but contain a normal nuclear genome. We observed normal steady-state levels of complex IV subunit II, as well as complex III core protein 2 and complex IV subunit IV in the cybrid cells (data not shown), confirming that the oxidative phosphorylation deficiency was recessive and of nuclear origin. 
To map the defective gene, we used microcellmediated chromosome transfer to identify a normal human chromosome that could functionally complement the biochemical defect in the fibroblasts from the index patient. ${ }^{4,6}$ We transferred all human autosomes and the $\mathrm{X}$ chromosome, one at a time, into an immortalized fibroblast line from the patient and used immunoblot analysis of complex IV subunit II as an assay for complementation. The protein levels of complex IV subunit II were restored only after the transfer of chromosome 3 (in 18 of 19 clones; data not shown).

Chromosomes incorporated into the genome of recipient cells by microcell-mediated chromosome transfer often undergo rearrangements and deletions. Deletion mapping of the donor chromosome in isolated clones can thus be used for fine mapping of the genomic region containing the complementing gene. Microsatellite mapping with a panel of polymorphic markers for chromosome 3 showed that one of the complementing clones contained only marker D3S1279, and that the fibroblast line from the patient was homozygous for markers D3S1279 and D3S1285. These data suggested that the candidate gene maps to the region located about $20 \mathrm{cM}$ from marker D3S1279 at 3q22-26.2 and flanked by markers D3S1569 and D3S1614.

A database search for candidate genes in this region identified two genes, EFG1 and MRPS22, that

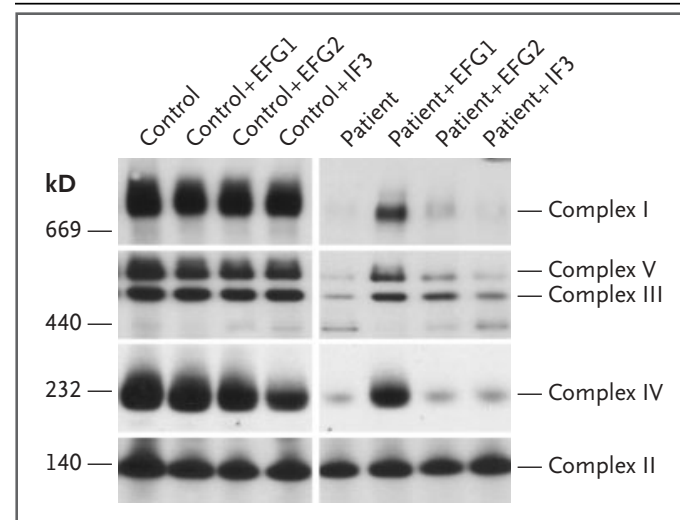

Figure 1. Analysis of Fibroblasts from the Index Patient. Fibroblasts from the index patient and a control were analyzed with the use of blue-native polyacrylamide-gel electrophoresis after transduction with retroviral constructs expressing mitochondrial translation factors. The gels were immunoblotted to assess the amount of fully assembled oxidative phosphorylation complexes. The expression of EFGl partially rescues the assembly defect in the patient's cells. code for proteins that are part of the mitochondrial translation machinery. ${ }^{11,12}$ Sequence analysis of cDNA showed a homozygous mutation (A521G) in the EFG1 cDNA isolated from the patient's immor-

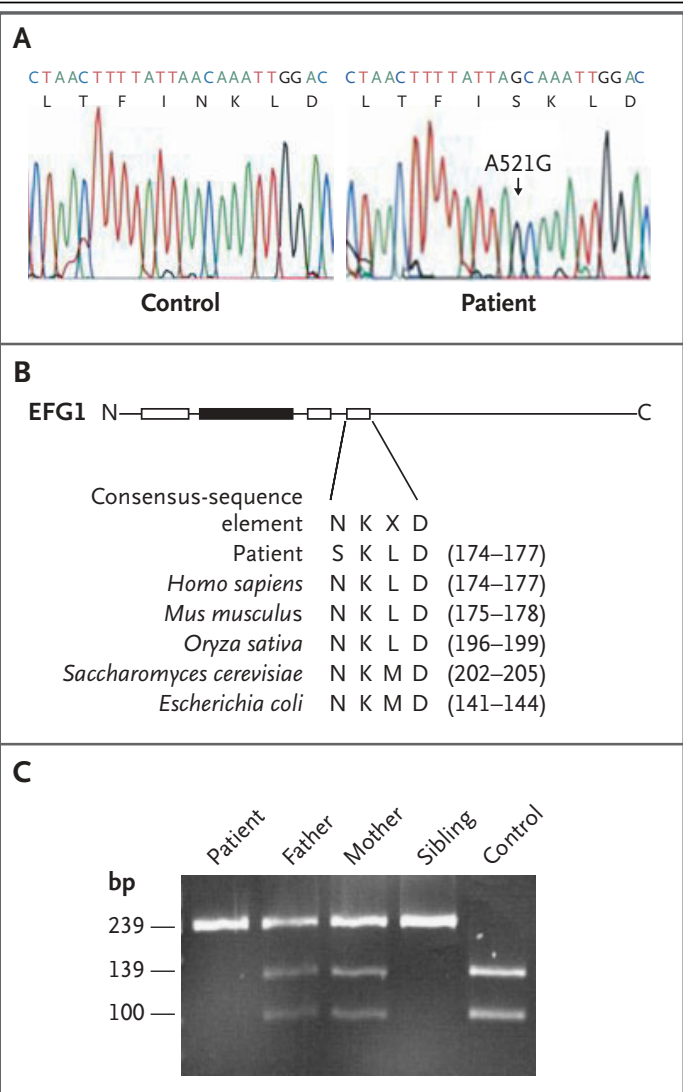

Figure 2. Analysis of EFG1.

Sequence analysis of the complementary DNA of EFG1 (Panel A) shows a homozygous A521G mutation in the patient as compared with the control. A schematic representation (Panel B) of the EFGl protein shows the GTPbinding elongation-factor signature sequence (black box) and the GTP-binding domain, consisting of three consensus-sequence elements with distinct spacing (white boxes). The consensus-sequence element NKXD is part of the GTP-binding domain and is responsible for base specificity. The consensus sequence of the GTPbinding domain is conserved across many functionally different families of GTP-binding proteins. The figure shows the absolute conservation of the sequence of asparagine at position 174 of the third consensus element in EFGl factor among taxa as far back as prokaryotes. Agarose gel was used in the analysis of the restrictionfragment-length polymorphisms that confirmed the mutation (Panel C). A polymerase-chain-reaction fragment of EFG1 encompassing the mutation was amplified from genomic DNA. The mutation eliminates an Msel restriction-enzyme site. Results from the patient, father, mother, sibling, and control are shown. 


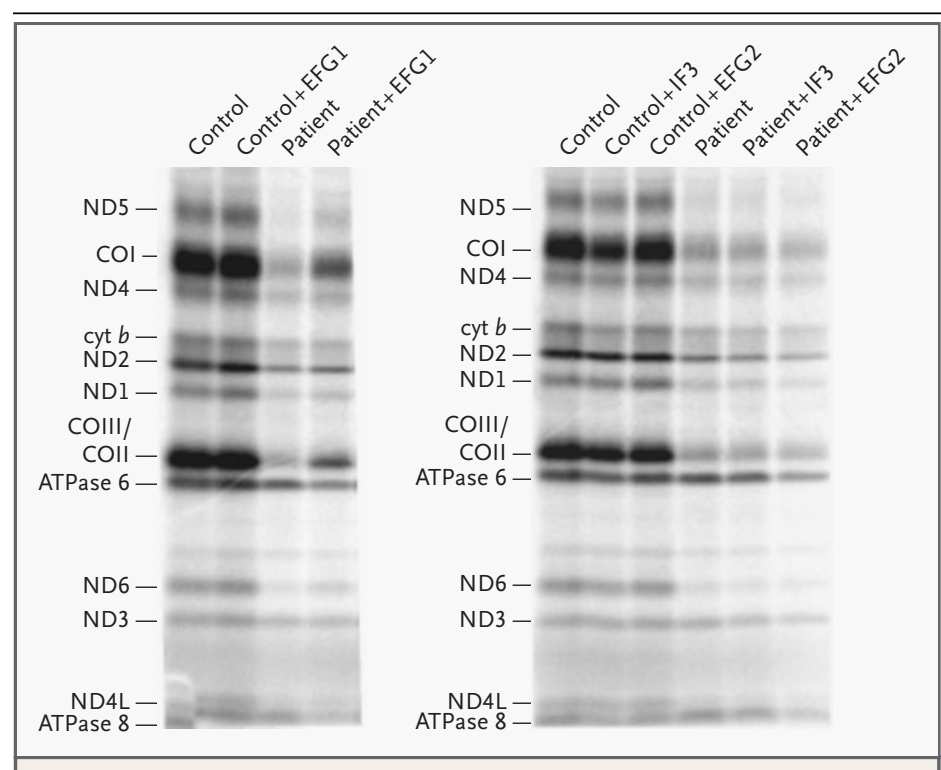

Figure 3. Analysis of Mitochondrial Translation Products.

Fibroblasts from the index patient that were overexpressing the mitochondrial translation factors EFGl, EFG2, and IF3 were pulse-labeled with [ ${ }^{35}$ S]methionine in the presence of an inhibitor of cytoplasmic protein synthesis (emetine) to assess the rate of mitochondrial translation. The patient's fibroblasts showed a severe defect in mitochondrial translation that overexpression of EFGl, as shown by Northern blotting, partially rectified (to about 40 percent of the expression observed in the controls) but that overexpression of the other translation factors did not affect. The proteins include seven subunits of complex I (ND), three subunits of complex IV (CO), the cytochrome $b$ subunit of complex III (cyt $b$ ), and two subunits of adenosine triphosphate (ATP) synthase (ATPase 6 and ATPase 8).

talized fibroblasts (Fig. 2A), which predicts an amino acid substitution of serine for asparagine (N174S) at a position that has been absolutely conserved during evolution (Fig. 2B). The presence of the mutation was confirmed in genomic DNA from the index patient and the sibling by analysis of restriction-fragment-length polymorphisms (Fig. 2C). The same analysis showed that both parents were heterozygous for the mutation. The mutation could not be detected in genomic DNA of 100 controls of Lebanese origin who were found to be homozygous for the wild-type allele by analysis of restriction-fragment-length polymorphisms. The index patient's cDNA sequence of MRPS22 was normal.

Pulse labeling of the mitochondrial translation products with $\left.{ }^{35} \mathrm{~S}\right]$ methionine showed a decrease in overall mitochondrial translation to about 20 percent of the overall translation in the controls. The deficit in translation varied from protein to protein. For instance, the expression of complex IV subunits I, II, and III was reduced to about 15 percent of the expression of the complex IV subunits in the controls, and expression of ND5 and ND6 to about 10 percent of control equivalents, whereas the other ND subunits were less affected (30 to 60 percent of control equivalents). Overexpression of EFG1 cDNA in fibroblasts from the index patient increased the overall mitochondrial translation to about 40 percent of the expression observed in the controls (Fig. 3); overexpression of two other mitochondrial translation factors (EFG2 or IF3) had no effect on mitochondrial translation.

Rescue of mitochondrial translation in fibroblasts from the index patient was accompanied by a rescue of complex IV activity from 20 percent of the activity in the controls to 72 percent. Furthermore, we observed an increase in the amount of fully assembled complexes I, III, IV, and V (Fig. 1). Overexpression of EFG2 slightly increased the amount of fully assembled complexes I, III, and V but did not result in a substantial increase in complex IV activity.

\section{DISCUSSION}

Synthesis of mitochondrial proteins requires a number of initiation, elongation, and termination (or release) factors, all of which are encoded by nuclear genes. The cDNA for two initiation factors (IF2, IF3), ${ }^{13,14}$ four elongation factors (EFTu, ${ }^{15}$ EFTs, ${ }^{16}$ EFG $1,{ }^{17}$ and $\mathrm{EFG}^{3}$ ), and a release factor $(\mathrm{RF} 1)^{18}$ have been cloned and sequenced in human and other mammalian species. IF2 promotes the binding of formyl methionyl-transfer RNA (fMettRNA) to the small ribosomal subunit in the presence of GTP and a template, ${ }^{19}$ whereas IF3 promotes the dissociation of the two ribosomal subunits, which produces free small subunits for the initiation of translation. ${ }^{14}$ The mammalian elongation factor Tu participates in the formation of the ternary complex that includes EFTu, GTP, and aminoacyl-tRNA, which delivers the aminoacyl-tRNA to the acceptor site of the ribosome. ${ }^{20}$ The energy required for this process is supplied by the hydrolysis of GTP, which is followed by the release of EFTu from the ribosome as an EFTu-guanosine diphosphate (GDP) complex. The exchange of GDP for GTP, which regenerates EFTu-GTP, is accomplished by EFTs. ${ }^{20}$

Although the roles of the mammalian elongation factors G1 and G2 have not been studied, elongation factor $\mathrm{G}$ in prokaryotes hydrolyzes $\mathrm{GTP}^{21}$ 
and, in so doing, catalyzes the translocation of peptidyl-tRNA from the ribosomal-acceptor site to the peptidyl site after peptide-bond formation. Concomitant movement of mRNA exposes the next codon in the acceptor site. ${ }^{22}$ RF1 recognizes stop codons and promotes the release of the completed protein chain.

It is not clear why there are two mammalian homologues of the prokaryotic elongation factor $\mathrm{G}$. Perhaps each catalyzes translation from a specific set of mRNA, or perhaps they are redundant, to ensure survival when one fails. Studies of yeast lend support to the latter hypothesis; deletion mutations of either EFG1 or EFG2 are viable, although EFG1 mutations have a phenotype of respiratory deficiency. ${ }^{23}$ Our observations suggest that EFG1 is an essential gene and that EFG1 and EFG2 have different but overlapping roles in mitochondrial translation (Fig. 1). Although the expression of both proteins is ubiquitous, ${ }^{11,17}$ the proteins may have tissuespecific functions, consistent with the severe effect of mutant EFG1 in the liver and brain and the mild effect in the heart and muscles.

Many GTP-binding proteins contain three highly conserved consensus elements (Fig. 2B). ${ }^{24}$ The A521G mutation effects the substitution of a highly conserved asparagine residue present in the consensus-sequence element NKXD (where $\mathrm{X}$ indicates any amino acid), which is part of the GTP- binding domain and is responsible for base specificity. ${ }^{24}$ A mutant variant of another GTP-binding protein, Ras p21, with replacement of the equivalent asparagine by lysine or tyrosine, is unable to bind guanine nucleotides or to transform NIH 3T3 cells. ${ }^{25}$ The mutant residue in EFG1 may also blunt GTP-binding activity, with consequent loss of efficiency of mitochondrial translation.

We report a mutation affecting a nuclear-encoded component of the mitochondrial translation system in an oxidative phosphorylation disorder. Patients with combined deficiencies of oxidative phosphorylation enzymes are at least as common as those with isolated deficiencies of single-enzyme complexes. Nuclear genes encoding the mitochondrial translation factors are good candidates as causative genes in these cases.

Supported by the Prinses Beatrix Fonds (grants 98-0108 and MAR02-0104 to Professor Smeitink and Associate Professor Bert van den Heuvel) and by a grant from the Canadian Institutes for Health Research (to Dr. Shoubridge). Dr. Shoubridge is an international scholar of the Howard Hughes Medical Institute and a senior scientist of the Canadian Institutes for Health Research. Dr. Antonicka is supported by a postdoctoral fellowship from the Canadian Institutes for Health Research and Muscular Dystrophy Canada.

We are indebted to Ms. Jeanette Pots, Ms. Guy-Hellen Guercin, Ms. Heleen Diepstra, and Mr. Timothy Johns for technical assistance; to Professors David Thorburn (Melbourne, Australia), Arnold Munnich and Valerie Cormier-Dare (Paris), Guy Rouleau (Montreal), and Andre Megarbane (Beirut, Lebanon) for supplying us with the samples of anonymized control DNA; and to Professor Anne Lombes (Paris) for the kind gift of the anti-ND1 antibody.

\section{REFEREN CES}

1. SmeitinkJ, van den Heuvel L, DiMauro S. The genetics and pathology of oxidative phosphorylation. Nat Rev Genet 2001;2: 342-52.

2. Jacobs HT. Disorders of mitochondria protein synthesis. Hum Mol Genet 2003;12 R293-R301.

3. Lochmuller H, Johns $\mathrm{T}$, Shoubridge EA Expression of the E6 and E7 genes of human papillomavirus (HPV16) extends the life span of human myoblasts. Exp Cell Res 1999; 248:186-93.

4. Zhu Z, Yao J, Johns T, et al. SURF1, encoding a factor involved in the biogenesis of cytochrome c oxidase, is mutated in Leigh syndrome. Nat Genet 1998;20:337-43.

5. Rubio-Gozalbo ME, Ruitenbeek W, Bentlage HA, et al. Favourable clinical course in an infant with severe deficiency of complex III of the respiratory chain combined with less severe deficiencies of complexes I, II and IV. Eur J Pediatr 1997;156: 931-4.

6. Fournier RE. A general high-efficiency procedure for production of microcell hybrids. Proc Natl Acad Sci U S A 1981;78: 6349-53.

7. Cuthbert AP, Trott DA, Ekong RM, et al. Construction and characterization of a highly stable human: rodent monochromosoma hybrid panel for genetic complementation and genome mapping studies. Cytogenet Cell Genet 1995;71:68-76.

8. Schagger $H$, von Jagow $G$. Blue native electrophoresis for isolation of membrane protein complexes in enzymatically active form. Anal Biochem 1991;199:223-31.

9. Boulet L, Karpati G, Shoubridge EA. Distribution and threshold expression of the tRNA(Lys) mutation in skeletal muscle of patients with myoclonic epilepsy and raggedred fibers (MERRF). Am J Hum Genet 1992; 51:1187-200.

10. Ory DS, Neugeboren BA, Mulligan RC. A stable human-derived packaging cell line for production of high titer retrovirus/vesic ular stomatitis virus G pseudotypes. Proc Natl Acad Sci U S A 1996;93:11400-6.
11. Hammarsund M, Wilson W, Corcoran $\mathrm{M}$, et al. Identification and characterization of two novel human mitochondrial elongation factor genes, hEFG2 and hEFG1, phylogenetically conserved through evolution. Hum Genet 2001;109:542-50.

12. Kenmochi N, Suzuki T, Uechi T, et al. The human mitochondrial ribosomal protein genes: mapping of 54 genes to the chromosomes and implications for human disorders. Genomics 2001;77:65-70.

13. Ma L, Spremulli LL. Cloning and sequence analysis of the human mitochondrial translational initiation factor $2 \mathrm{cDNA}$. J Biol Chem 1995;270:1859-65.

14. Koc EC, Spremulli LL. Identification of mammalian mitochondrial translational initiation factor 3 and examination of its role in initiation complex formation with natural mRNAs. J Biol Chem 2002;277: 35541-9.

15. Ling M, Merante F, Chen HS, Duff C, Duncan AM, Robinson BH. The human mitochondrial elongation factor tu (EF-Tu) gene: 
cDNA sequence, genomic localization, ge- $\quad$ cycling factor. Biochim Biophys Acta 1998; nomic structure, and identification of a 1443:245-50.

pseudogene. Gene 1997;197:325-36.

16. Xin H, Woriax V, Burkhart W, Spremulli

LL. Cloning and expression of mitochondrial translational elongation factor Ts from bovine and human liver. J Biol Chem 1995 270:17243-9.

17. Gao J, Yu L, Zhang P, et al. Cloning and characterization of human and mouse mitochondrial elongation factor G, GFM and Gfm, and mapping of GFM to human chro mosome 3q25.1-q26.2. Genomics 2001;74 109-14.

18. Zhang Y, Spremulli LL. Identification and cloning of human mitochondrial translational release factor 1 and the ribosome re-
19. Ma J, Spremulli LL. Expression, purification, and mechanistic studies of bovine mitochondrial translational initiation fac-

20. Cai YC, Bullard JM, Thompson NL Spremulli LL. Interaction of mitochondria elongation factor Tu with aminoacyl-tRNA and elongation factor Ts. J Biol Chem 2000 275:20308-14.

21. Katunin VI, Savelsbergh A, Rodnina MV, Wintermeyer W. Coupling of GTP hydrolysis by elongation factor $\mathrm{G}$ to translocation and factor recycling on the ribosome. Biochemistry 2002;41:12806-12.

22. Rodnina MV, Stark H, Savelsbergh A, et tor 2. J Biol Chem 1996;271:5805-11. al. GTPases mechanisms and functions of translation factors on the ribosome. Biol Chem 2000;381:377-87.

23. Vambutas A, Ackerman SH, TzagoloffA. Mitochondrial translational-initiation and elongation factors in Saccharomyces cerevisiae. Eur J Biochem 1991;201:643-52.

24. Dever TE, Glynias MJ, Merrick WC. GTP-binding domain: three consensus sequence elements with distinct spacing. Proc Natl Acad Sci U S A 1987;84:1814-8.

25. Clanton DJ, Hattori S, Shih TY. Mutations of the ras gene product p21 that abolish guanine nucleotide binding. Proc Natl Acad Sci U S A 1986;83:5076-80.

Copyright @ 2004 Massachusetts Medical Society. 